\title{
Predictors of Permanent Pacemaker Implantation After Coronary Artery Bypass Grafting and Valve Surgery in Adult Patients in Current Surgical Era
}

\author{
Bandar Al-Ghamdia, b, d, Yaseen Mallawi ${ }^{\mathrm{a}}$, Azam Shafquat ${ }^{\mathrm{a}}$, , Alexandra Ledesma ${ }^{\mathrm{a}}$, \\ Nadiah AlRuwailia, Mohamed Shoukria, c, Shahid Khan ${ }^{\text {a }}$, Aly Al Sanei ${ }^{\mathrm{a}}$
}

\begin{abstract}
Background: Permanent pacemaker (PPM) implantation after cardiac surgery is required in $0.4-6 \%$ of patients depending on cardiac surgery type. PPM implantation in the early postoperative period may reduce morbidity and postoperative hospital stay. We performed a retrospective review of electronic medical records of adult patients with coronary artery bypass grafting $(\mathrm{CABG})$, valve surgery, or both, over a 3-year period. Our aim was to identify predictors of PPM requirements and PPM dependency on follow-up in the current surgical era.
\end{abstract}

Methods: After exclusion of patients with congenital heart disease, patients who already had a PPM or implantable cardioverter defibrillator (ICD), and patients with an indication for PPM or ICD before surgery, we identified 1,234 adult patients who underwent cardiac surgery between January 2007 and December 2009. A retrospective review of electronic medical records and pacemaker clinic data was performed.

Results: Patients' mean age was $46.65 \pm 16$ years, and $59 \%$ were males. CABG was performed in $575(46.6 \%)$ cases, aortic valve replacement in $263(21.3 \%)$, mitral valve replacement in $333(27 \%)$, and tricuspid valve replacement in 76 patients $(6.2 \%)$. Twenty patients $(1.6 \%)$ required implantation of a PPM postoperatively. Indications for PPM implantation included complete atrioventricular (AV) block in $13(65 \%)$, sick sinus syndrome in three $(15 \%)$, and atrial fibrillation (AF) with a slow ventricular rate in four (20\%). Predictors for PPM requirement by multivariate analysis were the presence of pulmonary hypertension (P-HTN), reoperation, and left bundle branch block (LBBB) $(\mathrm{P}<0.05)$. Late follow-up was available in 18 patients, at $84.5 \pm 30$ months. Eleven patients (61\%) were PPM

Manuscript accepted for publication July 21, 2016

${ }^{a}$ Heart Centre, King Faisal Specialist Hospital and Research Centre, Riyadh, Saudi Arabia

${ }^{\mathrm{b} C}$ College of Medicine, Alfaisal University, Riyadh, Saudi Arabia

${ }^{c}$ National Biotechnology Center, King Faisal Specialist Hospital and Research Centre, Riyadh, Saudi Arabia

${ }^{\mathrm{d}}$ Corresponding Author: Bandar Al-Ghamdi, Heart Centre, King Faisal Specialist Hospital and Research Centre, MBC-16, PO Box 3354, Riyadh 11211, Saudi Arabia. Email: balghamdi@kfshrc.edu.sa

doi: http://dx.doi.org/10.14740/cr480w dependent on long-term follow-up.

Conclusions: Patients at high risk for PPM implantation after cardiac surgery include those with P-HTN, reoperation, and pre-existing LBBB. Of those receiving a PPM, about one-third will recover at least partially at long-term follow-up. We recommend preoperative assessment for risk of requiring postoperative PPM, to counsel patients about this risk and early PPM implantation in high-risk patients who are PPM dependent after surgery.

Keywords: Pacemaker; Cardiac surgery; Conduction system

\section{Introduction}

Permanent pacemaker (PPM) implantation after cardiac surgery is required in $0.4-6 \%$ of patients depending on cardiac surgery type [1-14].

In recent times, the incidence of postoperative PPM implantation has decreased due to improvements in surgical techniques, technological innovations and enhanced understanding of the mechanisms of injury, which generate the arrhythmia [15]. On the other hand, some studies have shown an increased incidence of PPM implantation after cardiac surgery after the year 2000 [16].

Identifying the patients who are at high risk for postoperative PPM implantation is important as it may reduce morbidity and postoperative hospital stay [12]. Several predictors of postoperative PPM implantations have been studied and observed; however, the risk factors for PPM implantation have been inconsistent across various studies. Our research aim was to determine the incidence and predictors for increased risk of postoperative PPM implantation in coronary artery bypass surgery $(\mathrm{CABG})$ and valve replacement surgeries.

\section{Materials and Methods}

We performed a retrospective chart review of 1,234 patients who underwent $\mathrm{CABG}$, valve replacement surgery, or both at the Heart Centre, King Faisal Specialist Hospital and Research Centre between January 2007 and December 2009. 
Table 1. Preoperative Data

\begin{tabular}{|c|c|c|c|}
\hline & PPM, no. (\%) & Non-PPM, no. (\%) & P value \\
\hline $\mathrm{N}$ & 20 & 1,214 & \\
\hline \multicolumn{4}{|l|}{ Demographic data } \\
\hline Age & $41.6 \pm 15.6$ & $51.3 \pm 16.4$ & 0.009 \\
\hline Age $>65$ years & $2(10 \%)$ & $279(23 \%)$ & 0.170 \\
\hline Gender (Male) & $11(55.0 \%)$ & $721(59.4 \%)$ & 0.692 \\
\hline Hypertension & $6.0(30 \%)$ & $576(47.4 \%)$ & 0.121 \\
\hline Diabetes & $7.0(35 \%)$ & $522(43 \%)$ & 0.473 \\
\hline Dyslipidemia & $0.0(0 \%)$ & $333(27.4 \%)$ & 0.006 \\
\hline Pulmonary hypertension & $12(60 \%)$ & $240(19.8 \%)$ & 0.000 \\
\hline Peripheral vascular disease & $1.0(5 \%)$ & $25(2.1 \%)$ & 0.364 \\
\hline Old CVA & $1.0(5 \%)$ & $42(3.5 \%)$ & 0.709 \\
\hline Renal impairment (creatinine $>1.5$ ) & $1.0(5 \%)$ & $116(9.6 \%)$ & 0.490 \\
\hline \multicolumn{4}{|l|}{ LV systolic function (LVEF) } \\
\hline Normal > 55\% & $0.0(0 \%)$ & $57(4.7 \%)$ & 0.321 \\
\hline Mildly impaired 45-55\% & $0.0(0 \%)$ & $98(8.1 \%)$ & 0.185 \\
\hline Moderately impaired 35-44\% & $4.0(20 \%)$ & $187(15.4 \%)$ & 0.573 \\
\hline Moderately to severely impaired $25-34 \%$ & $10(50 \%)$ & $449(37 \%)$ & 0.232 \\
\hline Severely impaired $<25 \%$ & $6.0(30 \%)$ & $419(34.5 \%)$ & 0.673 \\
\hline \multicolumn{4}{|l|}{ Coronary artery disease } \\
\hline Left main stenosis $(>50 \%)$ & $0(0 \%)$ & $168(13.8 \%)$ & 0.072 \\
\hline Proximal LAD stenosis (> 70\%) & $1(5 \%)$ & $520(42.8 \%)$ & 0.001 \\
\hline LCX & $1(5 \%)$ & $453(37.3 \%)$ & 0.003 \\
\hline $\mathrm{RCA}$ & $3(15 \%)$ & $460(37.9 \%)$ & 0.036 \\
\hline \multicolumn{4}{|l|}{ Drugs } \\
\hline$\beta$-blockers & $9(45 \%)$ & $543(44.7 \%)$ & 0.981 \\
\hline Calcium channel blockers & $0(0 \%)$ & $51(4.2 \%)$ & 0.796 \\
\hline Digoxin & $1(5 \%)$ & $47(3.9 \%)$ & 0.796 \\
\hline Antiarrhythmic & $0(0 \%)$ & $9(0.7 \%)$ & 0.699 \\
\hline
\end{tabular}

PPM: permanent pacemaker; non-PPM: non-permanent pacemaker; CVA: cerebrovascular accident; LV: left ventricle; EF: ejection fraction; LAD: left anterior descending artery; LCX: left circumflex; RCA: right coronary artery.

Inclusion criteria consisted of all $\mathrm{CABG}$, valve replacement surgery, or both. Twenty patients received PPM within 90 days after cardiac surgery. The 1,214 remaining patients served as controls.

The demographic, clinical, preoperative, operative, and early postoperative data were obtained from the cardiac surgery database at our Heart Centre. This prospective database is part of an ongoing database of patients undergoing cardiac surgery at our center.

The perioperative electrocardiograms (ECGs) were collected routinely in the MUSE Cardiology Information System (GE Healthcare) which is the ECG database management system in our hospital. The ECG data of patients receiving a PPM were compared to those of the control group.

Patients with an indication for pacemaker implantation before the cardiac surgery and patients who underwent post- operative implantable cardioverter defibrillator (ICD) implantation who did not have indication for the permanent pacing were excluded from the study.

Data from pacemaker clinic follow-up visits were used to determine pacemaker dependency. The underlying rhythm was obtained by programming the pacemaker to VVI rate of 40 beats per minute during the patient's visit to the pacemaker outpatient clinic. Pacemaker dependency was defined as continuous pacing with lowering pacemaker rate to 40 beats per minutes for at least $10 \mathrm{~s}$ [12].

\section{Statistical analysis}

Descriptive statistics for the continuous variables were reported as mean \pm standard deviation and categorical variables were 
Table 2. Operative Data

\begin{tabular}{llll}
\hline & PPM & Non-PPM & P value \\
\hline $\mathrm{N}$ & 20 & 1,214 & \\
Elective & $19(95 \%)$ & $1,125(92.7 \%)$ & 0.691 \\
Emergency & $1.0(5 \%)$ & $89(7.3 \%)$ & 0.691 \\
Reoperation & $12(60 \%)$ & $301(24.9 \%)$ & 0.000 \\
CABG alone & $3.0(15 \%)$ & $572(47.2 \%)$ & 0.004 \\
Valve alone & $19(95 \%)$ & $703(58.1 \%)$ & 0.001 \\
Combined & $2(10 \%)$ & $123(10.2 \%)$ & 0.478 \\
MVR & $10(50 \%)$ & $323(26.6 \%)$ & 0.001 \\
AVR & $6.0(30 \%)$ & $257(21.2 \%)$ & 0.339 \\
TVR & $7.0(35 \%)$ & $69(5.7 \%)$ & 0.000 \\
PVR & $0.0(0 \%)$ & $16(1.3 \%)$ & 0.605 \\
BPT (min) & $169.8 \pm 77.2$ & $125.3 \pm 66.5$ & 0.003 \\
XCT (min) & $125 \pm 65.3$ & $89 \pm 48$ & 0.001 \\
XCT $>120$ min & $3.3 \pm 2$ & $3 \pm 2$ & 0.622 \\
Minimum temperature $\left({ }^{\circ} \mathrm{C}\right)$ & $32 \pm 1.7$ & $32 \pm 4.8$ & 0.687 \\
Cold cardioplegia & $2(10 \%)$ & $196(16.1 \%)$ & 0.458 \\
\hline
\end{tabular}

PPM: permanent pacemaker; non-PPM: non-permanent pacemaker; CABG: coronary artery bypass grafting; AVR: aortic valve replacement; MVR: mitral valve replacement/repair; AVR: aortic valve replacement/ repair; TVR: tricuspid valve replacement/repair; PVR: pulmonic valve replacement/repair; BPT: cardiopulmonary bypass time; XCT: aortic cross-clamp time.

summarized as frequencies and percentages. The continuous variables of the two groups of patients were compared by Student's independent $t$-test, while the categorical variables were compared by Chi-square test. Logistic multivariate regression analysis was used to define the major predictors of requiring PPM postoperatively. The statistical level of significance was set at $\mathrm{P}<0.05$.

\section{Ethical consideration}

The study was conducted in accordance with the Helsinki Declaration as revised in 2013 and it was approved by research ethics board (REB) in our hospital prior to data collection and analysis.

\section{Results}

Patients' age was $46.65 \pm 16$ years, and $59 \%$ were males. CABG was performed in $575(46.6 \%)$ cases, aortic valve replacement in $263(21.3 \%)$, mitral valve replacement in 333 $(27 \%)$, and tricuspid valve replacement in 76 patients $(6.2 \%)$ (Tables 1 and 2 ). Twenty patients $(1.6 \%)$ required implantation of a PPM postoperatively. Indications for PPM implantation included complete atrioventricular (AV) block in 13 patients $(65 \%)$, sick sinus syndrome with symptomatic bradycardia in three $(15 \%)$, and atrial fibrillation (AF) with a slow ventricular rate in four $(20 \%)$ (Fig. 1). The types of cardiac surgery in PPM patients are shown in Figures 2 and 3. The timing of pacemaker implantation ranged from 7 to 73 days after surgery, with a mean of $17.65 \pm 14$ and median of 13.5 days. The delay in implantation in two patients was due to prolonged stay in the intensive care unit (ICU) and active infection. Two patients were discharged with junctional rhythm because they did not initially accept PPM implantation. Later on, when they presented for follow-up with no improvement in their rhythm, these patients did undergo PPM implantation. With exclusion of these two patients who were implanted on another admission, the mean time to PPM implantation was $14.89 \pm 5.4$ days, and median 13.5 days.

Table 1 shows the preoperative clinical profile of PPM patients compared with non-PPM patients. Patients with PPM were younger than those who did not require PPM. Dyslipidemia was more frequent in the non-PPM group. There were no significant differences in gender, hypertension, diabetes mellitus, peripheral vascular disease, old cerebrovascular accidents (CVAs), and left ventricular ejection fraction between the two groups. Pulmonary hypertension (P-HTN) was more prevalent in PPM patients. The presence of coronary artery disease (left anterior descending artery (LAD), left circumflex (LCX), and right coronary artery (RCA)) was seen more in the non-PPM group. The use of beta-blockers, calcium channel blockers, digoxin, and antiarrhythmic medications was equal in the two groups.

Most of the surgeries (95\%) were done on an elective basis. Reoperation surgeries were performed in 313 patients (25.3\%) (Table 2).

Table 3 shows the preoperative ECG characteristics of the patients. Patients requiring PPM had a higher incidence of 


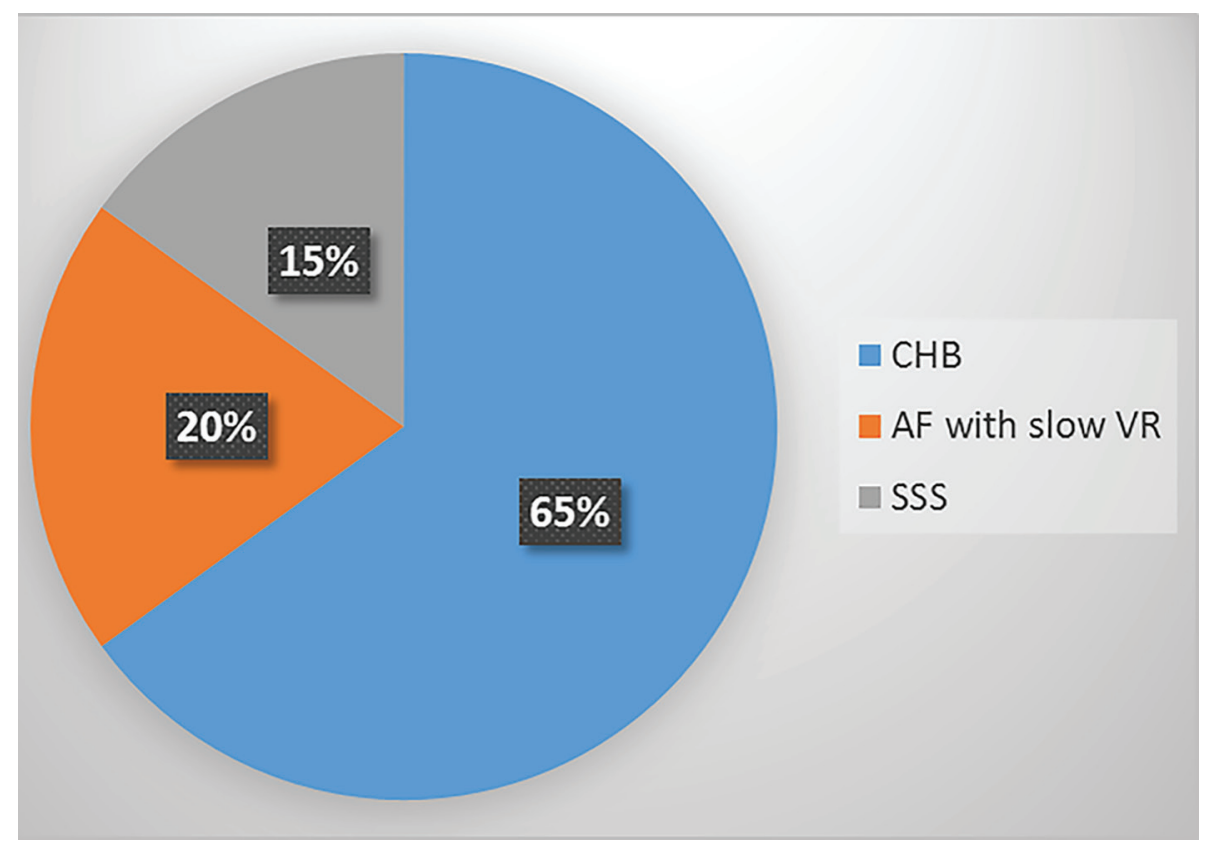

Figure 1. Indications of pacemaker. CHB: complete heart block; AF: atrial fibrillation; VR: ventricular rate; SSS: sick sinus syndrome.

preoperative conduction disorders in the form of left bundle branch block (LBBB), and first-degree AV block.

Patients requiring PPM had a longer overall postoperative hospitalization, but not necessarily a longer ICU stay. There was no difference in mortality between the two groups (Table 4).

Predictors for PPM requirement by multivariate analysis were the presence of P-HTN, reoperation surgery, and LBBB $(\mathrm{P}<0.05)$ (Table 5).

Long-term follow-up was available in 18 pacemaker patients with follow-up periods ranging from 35 to 109 months and a mean of $84.5 \pm 30$ months. Eleven patients $(61 \%)$ were PPM dependent.

\section{Discussion}

The requirement of PPM in our series was 1.6\%, which is consistent with what has been reported previously $[3,12]$.

The predictors for PPM post-cardiac surgery will be discussed in the order of preoperative, operative, and postopera-

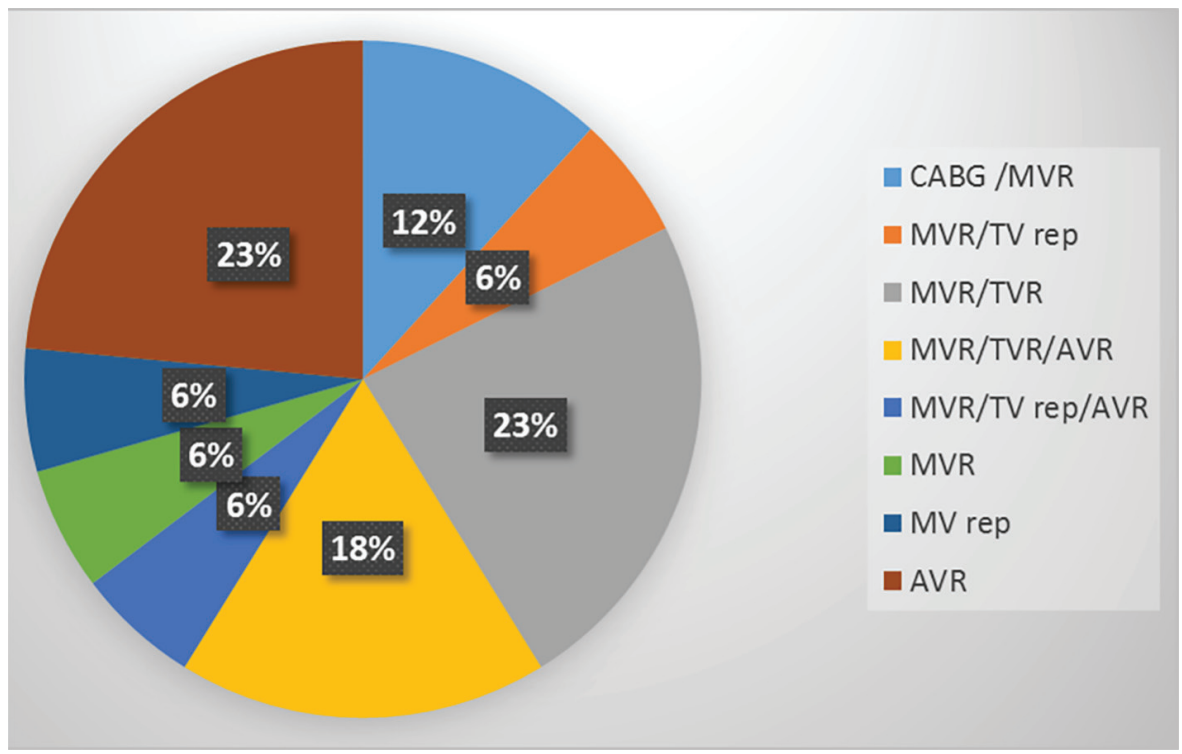

Figure 2. Type of surgery in the patients with permanent pacemaker. CABG: coronary artery bypass surgery; MV: mitral valve; TV: tricuspid valve; AV: aortic valve; R: replacement; rep: repair. 


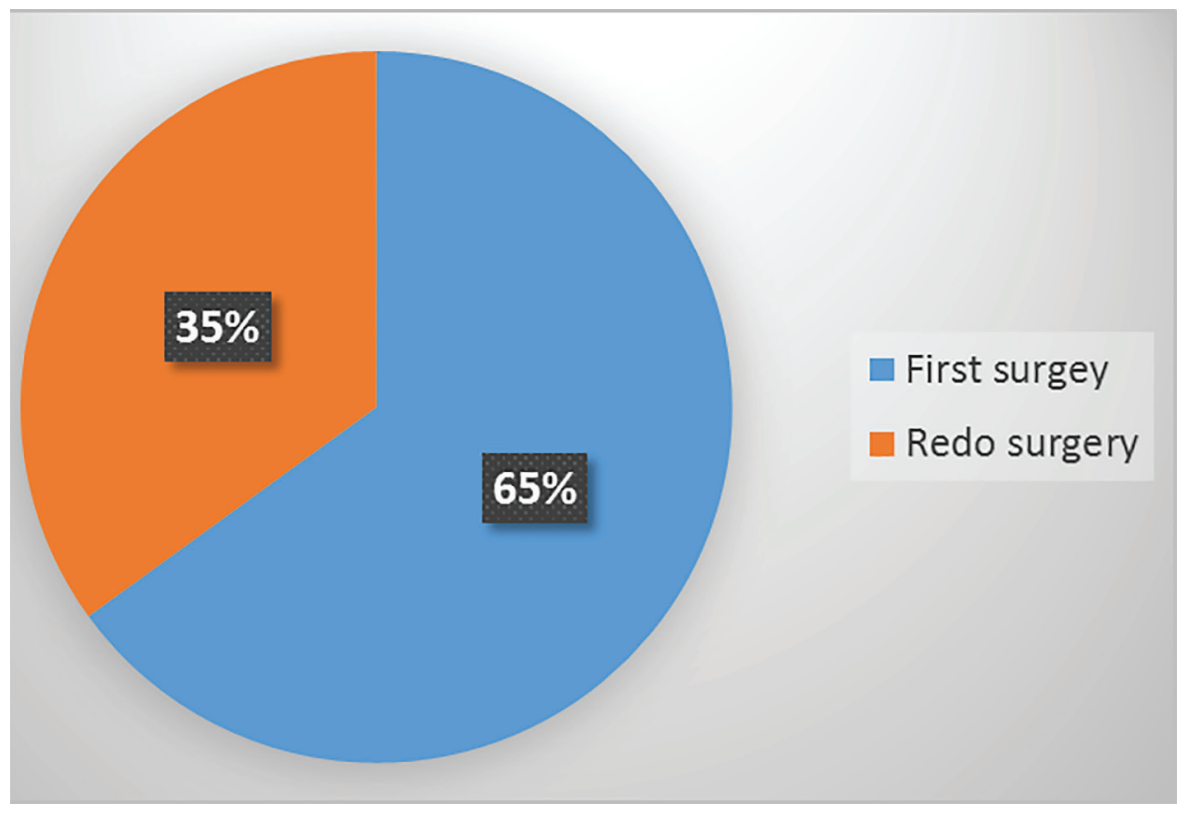

Figure 3. First vs. redo surgery in patients with permanent pacemakers.

tive periods.

\section{Preoperative predictors}

Our patients were younger than what has been reported in previous studies [1-14] due to valve surgery for young patients with rheumatic heart disease.

In our study, there was no difference in gender between the PPM and the non-PPM groups, unlike some previous studies which showed female gender as a predictor for PPM implantation $[3,5,8]$.

Age above 75 years at the time of surgery was reported previously as a risk factor for requiring a PPM [1, 3, 4, 13, $16,17]$, but this was not the case in our patients. Patients with P-HTN were found to have a higher risk of requiring PPM postoperatively. This was also noted in one previous study [7].

The use of rate lowering cardiac medications (e.g., betablockers, calcium channel blockers, digoxin, and anti-arrhythmic medications) is one of the suggested mechanisms contributing to conduction system damage postoperatively. Some studies showed that preoperative use of antiarrhythmic medications, like digoxin [7], calcium channel blockers [8], amiodarone and sotalol [11], increased the risk for PPM postoperatively, but we found no relation between preoperative medications and the requirement of PPM in our patients.

The presence of conduction system disease preoperatively $[1,7]$, right bundle branch block (RBBB) or LBBB [8, 14], LBBB alone [2, 12], RBBB alone [13, 17], first-degree AV block [14] or left anterior fascicular block (LAFB) [14] was found to be a predictor for requiring PPM postoperatively. In our patients, LBBB was found to be a significant predictor. Preoperative non-sinus rhythm $[3,18]$ or AF [5] were risk factors in some previous studies but not in our series.

The cardiac conduction system is susceptible to damage during cardiac surgery. The main physiopathological mechanisms involved in the development of cardiac conduction disorders postoperatively are myocardial ischemia, inadequate cardiac protection during surgery, and direct surgical injury [15]. Ischemia may be more common in patients with left main

Table 3. Preoperative Electrocardiogram (ECG)

\begin{tabular}{llll}
\hline & PPM, no. (\%) & Non-PPM, no (\%) & P value \\
\hline N & 20 & 1,214 & \\
AFIB & $5(25 \%)$ & $179(14.7 \%)$ & 0.202 \\
Any BBB & $3(15 \%)$ & $91(7.5 \%)$ & 0.213 \\
LBBB & $2(10 \%)$ & $28(2.3 \%)$ & 0.027 \\
RBBB & $2(10 \%)$ & $64(5.3 \%)$ & 0.351 \\
First-degree AVB & $4(20 \%)$ & $66(5.4 \%)$ & 0.005 \\
\hline
\end{tabular}

PPM: permanent pacemaker; non-PPM: non-permanent pacemaker; AF: atrial fibrillation; BBB: bundle branch block; LBBB: left bundle branch block; RBBB: right bundle branch block; AVB: atrioventricular block. 
Table 4. Postoperative Data

\begin{tabular}{llll}
\hline & $\begin{array}{l}\text { PPM, } \\
\text { no }(\%)\end{array}$ & $\begin{array}{l}\text { Non-PPM, } \\
\text { no (\%) }\end{array}$ & P value \\
\hline $\mathrm{N}$ & 20 & 1,214 & \\
Perioperative MI & $0.0(0 \%)$ & $267(22 \%)$ & 0.018 \\
Elevated Trop-T $(>0.01)$ & $8.0(40 \%)$ & $689(56.8 \%)$ & 0.134 \\
ICU stay (days) & $6.80 \pm 6.0$ & $9 \pm 4.74$ & 0.311 \\
Hospital stay (days) & $18.1 \pm 11.5$ & $12.3 \pm 8.5$ & 0.000 \\
Mortality & $0(0.0 \%)$ & $92(7.6 \%)$ & 0.201 \\
\hline
\end{tabular}

PPM: permanent pacemaker; non-PPM: non-permanent pacemaker; MI: myocardial infarction; Trop-T: troponin-T; ICU: intensive care unit.

or proximal LAD disease $[19,20]$ but this was not the case in our patients.

\section{Operative predictors}

Mechanical trauma to the conduction system arising secondary to valve operation, or other surgeries close to the AV node, is another risk factor for AV node conduction disorder [15]. The need for PPM postoperatively is more common in valve surgeries especially aortic, mitral and tricuspid valve surgeries $[1,3,4,9,12,16,18]$. We found that of all valve surgeries in general, mitral valve and tricuspid valve surgeries had a higher risk of requiring PPM, but with multivariate analysis, these did not emerge as independent risk factors.

In addition to coronary artery disease, ischemic injury of the sinus node or other parts of the conduction system might occur during any cardiac procedure because of inadequate myocardial protection during surgery [15].

Prolonged cardiopulmonary bypass time and cross-clamp time have been found in this study as well as in previous studies $[8,11,16,18]$ to be associated with postoperative PPM requirement. Cold blood cardioplegia was also found to be a risk factor in previous studies [3-5], but not in our current study.

About $35 \%$ of PPM patients in this study were undergoing redo surgery. Reoperation was found in this study to be a significant risk as it was in previous studies [3-5, 18].

\section{Postoperative predictors}

Postoperative conduction disturbances [7] and high-grade AV block [6] were found to increase risk of PPM postoperatively. Most of our patients who required PPM had postoperative complete heart block (CHB).

In our patients, time to pacemaker implant was longer than what has been reported previously [21] because we enrolled patients with pacemaker implantation up to 3 months postoperatively.

About $61 \%$ of our patients were pacer dependent on longterm follow-up, which is similar to what has been reported before $[12,22]$.

From a practical point of view, the decision regarding
Table 5. Predictors of Permanent Pacemaker Implantation

\begin{tabular}{lllll} 
Variables & P value & OR & \multicolumn{2}{c}{$\begin{array}{c}\text { 95\% confidence interval of } \\
\text { the difference }\end{array}$} \\
\cline { 4 - 5 } & & & Lower & Upper \\
\hline LBBB & 0.042 & 2.0 & 1.32 & 5.54 \\
P-HTN & 0.001 & 5.08 & 1.941 & 13.296 \\
Reoperation & 0.001 & 4.515 & 1.828 & 11.15 \\
\hline
\end{tabular}

LBB: left buddle branch block; P-HTN: pulmonary hypertension.

which patient will require PPM and when to implant the device are the most important clinical questions. According to the American College of Cardiology, the American Heart Association and Heart Rhythm Society guidelines, PPM implantation is indicated for third-degree and advanced second-degree AV block, at any anatomic level, associated with postoperative AV block which is not expected to resolve after cardiac surgery (level of evidence: C) [23].

The decision about PPM implantation and its timing is left for treating physicians' discretion. We believe that patients with high-risk factors need to be counseled preoperatively about their risk of requiring PPM postoperatively. Additionally, implantation of the pacemaker early after cardiac surgery is recommended for conduction system disorders which are unlikely to recover. Placing epicardial PPM leads in identified at-risk patients during the surgery, especially with tricuspid valve intervention, may also be helpful.

\section{Limitations of the study}

The main limitation in this study is the small number of patients who underwent PPM implantation after cardiac surgery. As a retrospective analysis, the study suffers from a number of limitations that should be considered when interpreting the results: the absence of a prospective validation of the model, the lack of sufficient information on annular calcification and root dilatation, operative data such as root replacement, and extent of perioperative annular debridement, all of which have been identified in earlier studies as predictors of PPM implantation [18].

\section{Conclusion}

Patients at high risk for PPM implantation after cardiac surgery include those with the presence of P-HTN, reoperation surgeries, and LBBB. Of those receiving a PPM, about $40 \%$ will recover at least partially at long-term follow-up. We recommend preoperative assessment for risk of requiring PPM postoperatively to counsel patients about this risk and early PPM implantation in high-risk patients who are dependent on temporary pacemaker after surgery.

\section{Grant Support}

None. 


\section{Financial Disclosures}

No source of funding to declare.

\section{Competing Interests}

The authors declare that they have no competing interests.

\section{References}

1. Goldman BS, Hill TJ, Weisel RD, Scully HE, Mickleborough LL, Pym J, Baird RJ. Permanent cardiac pacing after open-heart surgery: acquired heart disease. Pacing Clin Electrophysiol. 1984;7(3 Pt 1):367-371.

2. Emlein G, Huang SK, Pires LA, Rofino K, Okike ON, Vander Salm TJ. Prolonged bradyarrhythmias after isolated coronary artery bypass graft surgery. Am Heart J. 1993;126(5):1084-1090.

3. Del Rizzo DF, Nishimura S, Lau C, Sever J, Goldman BS. Cardiac pacing following surgery for acquired heart disease. J Card Surg. 1996;11(5):332-340.

4. Gordon RS, Ivanov J, Cohen G, Ralph-Edwards AL. Permanent cardiac pacing after a cardiac operation: predicting the use of permanent pacemakers. Ann Thorac Surg. 1998;66(5):1698-1704.

5. Sachweh JS, Vazquez-Jimenez JF, Schondube FA, Daebritz SH, Dorge H, Muhler EG, Messmer BJ. Twenty years experience with pediatric pacing: epicardial and transvenous stimulation. Eur J Cardiothorac Surg. 2000;17(4):455-461.

6. Meimoun P, Zeghdi R, D'Attelis N, Berrebi A, Braunberger E, Deloche A, Fabiani JN, et al. Frequency, predictors, and consequences of atrioventricular block after mitral valve repair. Am J Cardiol. 2002;89(9):1062-1066.

7. Limongelli G, Ducceschi V, D'Andrea A, Renzulli A, Sarubbi B, De Feo M, Cerasuolo F, et al. Risk factors for pacemaker implantation following aortic valve replacement: a single centre experience. Heart. 2003;89(8):901-904.

8. Erdogan O, Augostini R, Saliba W, Juratli N, Wilkoff BL. Transiliac. Pacing Clin Electrophysiol. 2006;29:10551062.

9. Ben Ameur Y, Baraket F, Longo S, Annabi N, Bouraoui L, Mokni W, Battikh K, et al. [Conductive disorders following open-heart valvular surgery. Concerning 230 operated patients]. Ann Cardiol Angeiol (Paris). 2006;55(3):140143.

10. Imren Y, Benson AA, Oktar GL, Cheema FH, Comas G, Naseem $T$. Is use of temporary pacing wires following coronary bypass surgery really necessary? J Cardiovasc Surg (Torino). 2008;49(2):261-267.

11. Berdajs D, Schurr UP, Wagner A, Seifert B, Turina MI, Genoni M. Incidence and pathophysiology of atrioventricular block following mitral valve replacement and ring annuloplasty. Eur J Cardiothorac Surg. 2008;34(1):55-61.

12. Merin $\mathrm{O}$, Ilan M, Oren A, Fink D, Deeb M, Bitran D, Silberman S. Permanent pacemaker implantation follow- ing cardiac surgery: indications and long-term follow-up. Pacing Clin Electrophysiol. 2009;32(1):7-12.

13. Schurr UP, Berli J, Berdajs D, Hausler A, Dzemali O, Emmert M, Seifert B, et al. Incidence and risk factors for pacemaker implantation following aortic valve replacement. Interact Cardiovasc Thorac Surg. 2010;11(5):556560.

14. Nardi P, Pellegrino A, Scafuri A, Bellos K, De Propris S, Polisca P, Chiariello L. Permanent pacemaker implantation after isolated aortic valve replacement: incidence, risk factors and surgical technical aspects. J Cardiovase Med (Hagerstown). 2010;11(1):14-19.

15. Cardiac Pacemakers - Biological Aspects, Clinical Applications and Possible Complications. Edited by Mart Min, ISBN 978-953-307-639-3, 206 pages, Publisher: InTech, Chapters published September 06, 2011 under CC BYNC-SA 3.0 license.

16. Raza SS, Li JM, John R, Chen LY, Tholakanahalli VN, Mbai M, Adabag AS. Long-term mortality and pacing outcomes of patients with permanent pacemaker implantation after cardiac surgery. Pacing Clin Electrophysiol. 2011;34(3):331-338

17. Guthier J, Ayub B, Nawaz Y, Saif H, Malacoff RF, Weiss M, Phillips T, Wu JK, Singer R, Mehta SM, Szydlowski G, Freudenberger R. (2014, July 28). Incidence and Predictors of Permanent Pacemaker Implantation after Valve Surgery-A Single Center Experience. Poster presented at the International Academy of Cardiology, Annual Scientific Sessions 2014, Boston, MA.

18. Elahi M, Usmaan K. The bioprosthesis type and size influence the postoperative incidence of permanent pacemaker implantation in patients undergoing aortic valve surgery. J Interv Card Electrophysiol. 2006;15(2):113-118.

19. Mosseri M, Meir G, Lotan C, Hasin Y, Applebaum A, Rosenheck S, Shimon D, et al. Coronary pathology predicts conduction disturbances after coronary artery bypass grafting. Ann Thorac Surg. 1991;51(2):248-252.

20. Caspi J, Amar R, Elami A, Safadi T, Merin G. Frequency and significance of complete atrioventricular block after coronary artery bypass grafting. Am J Cardiol. 1989;63(9):526-529.

21. Steyers CM, 3rd, Khera R, Bhave P. Pacemaker Dependency after Cardiac Surgery: A Systematic Review of Current Evidence. PLoS One. 2015;10(10):e0140340.

22. Glikson M, Dearani JA, Hyberger LK, Schaff HV, Hammill SC, Hayes DL. Indications, effectiveness, and longterm dependency in permanent pacing after cardiac surgery. Am J Cardiol. 1997;80(10):1309-1313.

23. Epstein AE, DiMarco JP, Ellenbogen KA, Estes NA, 3rd, Freedman RA, Gettes LS, Gillinov AM, et al. ACC/ AHA/HRS 2008 Guidelines for Device-Based Therapy of Cardiac Rhythm Abnormalities: a report of the American College of Cardiology/American Heart Association Task Force on Practice Guidelines (Writing Committee to Revise the ACC/AHA/NASPE 2002 Guideline Update for Implantation of Cardiac Pacemakers and Antiarrhythmia Devices): developed in collaboration with the American Association for Thoracic Surgery and Society of Thoracic Surgeons. Circulation. 2008;117(21):e350-408. 